\section{Cahiers de Narratologie}

Analyse et théorie narratives

$10.2 \mid 2001$

La voix narrative

\title{
La polyphonie narrative dans Novela de Andrés Choz de José María Merino : un exemple de procédé de mise en abyme
}

\section{Sébastien Robreau}

\section{(2) OpenEdition}

Journals

Édition électronique

URL : http://journals.openedition.org/narratologie/10274

DOI : 10.4000/narratologie.10274

ISSN : 1765-307X

Éditeur

LIRCES

Édition imprimée

Date de publication : 1 janvier 2001

Pagination : 395-404

ISBN : 2914561032

ISSN : 0993-8516

Référence électronique

Sébastien Robreau, « La polyphonie narrative dans Novela de Andrés Choz de José María Merino : un exemple de procédé de mise en abyme », Cahiers de Narratologie [En ligne], 10.2 | 2001, mis en ligne le 01 janvier 2001, consulté le 11 juin 2020. URL : http://journals.openedition.org/narratologie/10274 ; DOI : https://doi.org/10.4000/narratologie.10274 


\title{
LA POLYPHONIE NARRATIVE DANS NOVELA DE ANDRÉS CHOZ DE JOSÉ MARÍA MERINO : UN EXEMPLE DE PROCÉDÉ DE MISE EN ABYME
}

\author{
Sébastien ROBREAU \\ Université de Rennes 2
}

Avant toute chose, il paraît essentiel de donner quelques précisions quant à l'auteur et à ce livre. José María Merino est un écrivain espagnol contemporain né en 1941. Il s'est récemment distingué par la publication d'une anthologie du conte espagnol ${ }^{1}$. Ayant commencé sa carrière en tant que poète, il se rend vite compte que la narration est son principal centre d'intérêt. Après avoir collaboré avec Juan Pedro Aparicio et Luis Mateo Díez à l'élaboration de théories littéraires sur le roman, notamment par le biais des œuvres d'un auteur apocryphe, Sabino Ordás, créé de toutes pièces par les trois auteurs, Merino publie sa première œuvre romanesque en 1976 : Novela de Andrés Choz 2 .

Le choix de ce roman comme objet d'étude dans la perspective de la polyphonie narrative s'est presque imposé de lui-même. Outre le fait que dans ce premier roman Merino intègre sa réflexion sur la littérature par divers procédés que nous étudierons plus loin, c'est l'histoire en elle-même qui invite le lecteur à réfléchir sur le(s) narrateur(s). Ce roman est aussi le premier de la littérature espagnole à annoncer clairement qu'il est un métarécit ${ }^{3}$.

${ }^{1}$ MERINO, José María, selección y prólogo, Cien años de cuentos (1898-1998). Antología del cuento español en castellano, Alfaguara, col. Alfaguara Extra, 1998.

2 MERINO, José María, Novela de Andrés Choz, Alfaguara Bolsillo, Alfaguara, 1993. Les citations ainsi que les références au roman dans ce travail, sont toutes faites à partir de cette édition.

3 J'entends par métarécit un texte qui parle d'autres textes (paratextualité telle que la définit Genette) mais qui surtout fait des 
L'histoire de Novela de Andrés Choz paraît assez simple au premier abord. Le personnage principal, Andrés Choz est écrivain. Son médecin lui annonce que ses jours sont comptés à cause de son cancer. L'écrivain décide alors de se retirer dans sa maison de campagne et de reprendre l'écriture d'un projet romanesque qui n'avait jamais abouti : rédiger les mémoires d'un extraterrestre qui aurait atterri en Espagne juste après la guerre civile. Tour à tour chien, colonne de poussière blanche et enfin être humain à part entière, Ons (c'est son nom) découvre ainsi notre planète.

Parallèlement à cette histoire racontée par Choz, le roman dresse la vie de ce dernier et fait notamment état de sa correspondance avec son éditeur El Gordo, de ses amours avec la jeune Teresa et de son suicide raté grâce à l'intervention d'Armando, le petit ami de Teresa, thésard en lettres. A la fin du roman, les deux histoires se croisent pour n'en former qu'une seule: Ons et Choz sont en fait la même personne.

Le roman propose un dédoublement dont le titre se fait clairement l'écho. Il est en effet à lui seul un programme narratif puisqu'il porte le nom de son propre genre ${ }^{4}$. Il y a donc là une première manifestation de mise en abyme : l'auteur annonce explicitement au lecteur que ce qu'il va lire est une fiction. Mais il précise aussi que le sujet de ce texte fictionnel sera l'écriture par la seule évocation du nom «novela » $(\text { roman } »)^{5}$.

Cependant, le dédoublement de récits (et par conséquent de narrateurs) n'en est qu'à son début. En effet, dans les premières pages du livre, Andrés Choz retrouve les

références à lui-même, c'est ce que Genette appelle la transtextualité à propos de l'architexte dans Palimpsestes.

${ }^{4}$ Novela de Andrés Choz se traduit par Roman d'Andrés Choz. (C'est moi qui souligne). Ce livre restant inédit en France (du moins à ma connaissance), je traduis les citations et les indique en note entre crochets.

5 Il paraît opportun de préciser ici que le complément du nom «novela ", "de Andrés Choz », peut s'interpréter de deux manières. Soit il s'agit du sujet de "novela » et auquel cas nous sommes face à un roman qui traite d'un roman écrit par Andrés Choz. Soit il s'agit du complément d'objet de "novela " et alors le roman porte sur Andrés Choz. Le lecteur se rend compte à la fin du livre qu'il s'agit en fait des deux. 
feuilles manuscrites de l'ébauche de son roman. Sur la première d'entre elles est écrit le titre suivant : « NOVELA DEL HERMANO ONS » (p. 19). Nous nous trouvons face à ce que Dällenbach appelle une réduplication à l'infini' ${ }^{6}$.

On constate donc que la réduplication ne s'opère pas que sur le plan formel mais aussi sur le plan architectural et structurel du texte : un narrateur omniscient extradiégétique prend en charge la narration de la vie d'Andrés Choz qui prend lui-même en charge (mais cette fois en tant que narrateur intradiégétique) celle de la vie d'Ons. Ce dernier récit est toujours à la troisième personne mais sous le seul point de vue de Ons qui consigne ses observations sur les modes de vie humains dans son journal de bord à l'intention de ses congénères. Merino se livre à un jeu de poupées gigognes où sont étroitement imbriqués les narrateurs ${ }^{7}$. C'est là la première manifestation de polyphonie narrative dans cette œuvre ${ }^{8}$.

Cependant, c'est au niveau de la structure que la polyphonie narrative nous est le plus montrée. Nous allons nous attacher à trois recours essentiels de Merino pour le démontrer: les différences typographiques, le jeu sur les

6 La réduplication à l'infini consiste en la répétition d'un même phénomène plusieurs fois. $\mathrm{Cf}$. DÄLLENBACH, Lucien, Le récit spéculaire. Essai sur la mise en abyme, Collection Poétique, Seuil, Paris, 1977, pp. 51-52.

7 Pour une meilleure compréhension, je propose ici un schéma. J'utiliserai désormais le nom de récit-cadre pour le roman en luimême et le nom de récit inséré pour le roman écrit par Choz.

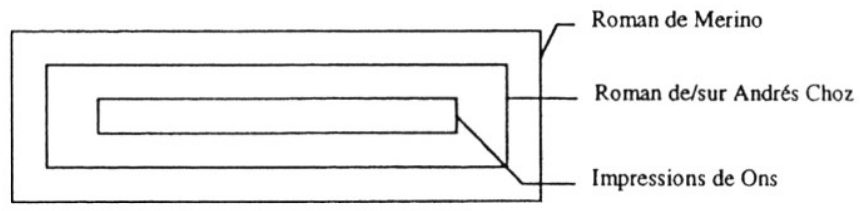

8 Même si la diégèse qui concerne Ons est rédigée à la troisième personne, la focalisation est bien souvent interne, au point qu'à un moment donné, la Machine qui sert de vaisseau spatial à l'extraterrestre, prend elle-même la parole: "Fin de Transmisión Excepcional »(p. 88). 
personnes verbales ainsi que sur le style ${ }^{9}$ et le discours réflexif sur le producteur et le récepteur du texte littéraire.

Les deux premiers chapitres sont certainement les plus représentatifs des changements typographiques voulus par l'auteur ${ }^{10}$. Il existe quatre typographies différentes. La première (que nous appellerons principale) représente à la fois le narrateur omniscient et Andrés Choz et est la plus fréquente dans le texte. La seconde, qui apparaît entre parenthèses, représente la voix du narrateur omniscient qui donne le passé de l'histoire par rapport au plan temporel de référence $^{11}$. La troisième consiste en des citations faites par $\mathrm{Choz}$ de son propre roman. Celles-ci apparaissent alors sous forme de caractères plus petits précédés de deux points. Enfin, les caractères en italique se réfèrent plutôt à des éléments culturels qui se rattachent de près ou de loin à Andrés $\mathrm{Choz}^{12}$.

Ces différences typographiques tendent à dérouter le lecteur. En effet, le roman s'ouvre sur la seconde typographie et nous force à nous demander tout au long de la première page, qui est le narrateur. Elle laisse ensuite place à la typographie principale qui retranscrit sans aucune présentation des protagonistes, un dialogue. A cela il faut ajouter l'organisation des chapitres qui elle aussi cherche à perdre le lecteur. Après un premier chapitre sans titre, apparaît un mystérieux «(Uno)» (p. 25), lui-même suivi d'un «CAPÍTULO PRIMERO » (p. 39). Le lecteur comprend ensuite que la

9 J'entends ici style au sens grammatical du terme.

10 Le plus flagrant est celui de l'alternance des chapitres: en minuscule et entre parenthèses, tous ceux qui concernent Andrés Choz ; en majuscules et précisant "CAPÍTULO ", ceux consacrés à Ons. L'alternance s'arrête avec le dernier ou les deux typographies sont réunies.

${ }^{11}$ Le récit commence in medias res par un dialogue entre Choz et son éditeur, au sujet de sa maladie. Le narrateur omniscient nous décrit alors les réactions de Choz juste après que son médecin lui ait annoncé son cancer.

12 Ils servent de fait pour une définition du dictionnaire (p. 13), pour un poème dont se souvient $\mathrm{Choz}$ (p.15), pour un poème écrit par lui-même (pp. 57-58), pour des notes au début d'un chapitre de son roman (p. 61), ou encore pour des mots sur lesquels il insiste lors d'une conversation avec Armando (p. 78) 
première typographie est réservée aux chapitres sur $\mathrm{Choz}$ alors que la deuxième correspond au roman écrit par celui-ci. Commence alors un jeu avec le lecteur qui n'en est qu'à son début.

En effet, Merino s'amuse tout au long du roman à intensifier et à rendre plus complexe l'utilisation des voix narratives, notamment par l'utilisation des personnes grammaticales. Au début du livre, si la typographie est déroutante, le narrateur quant à lui est rassurant en tant qu'omniscient ${ }^{13}$. Les cinq premiers chapitres distinguent clairement les voix narratives : le narrateur omniscient du récit-cadre (que nous appellerons $\mathrm{N} 1$ ) alterne avec la voix d'Andrés Choz qui apparaît dans ses lettres (A1) et le même Choz en tant qu'écrivain et utilisant une narration omnisciente pour Ons (N2). Le sixième chapitre amorce la complexité et la polyphonie : Al et $\mathrm{N} 1$ alternent à l'intérieur de ce même chapitre. De plus, N1 joue sur deux plans temporels qui se dédoublent et se croisent : la première et la deuxième rencontre entre $\mathrm{Choz}$ et Teresa. La dernière page du chapitre marque une première rupture chez N1 qui tutoie son personnage ${ }^{14}$.

Ce sont ensuite les points de vue qui sont imbriqués sans autre signe de distinction qu'une ligne blanche : alternance des points de vue de Choz et de Teresa au chapitre «(Seis)» (pp. 117-128) ; point de vue de Teresa qui, dans son lit, revit une de ses rencontres avec Choz en même temps qu'elle observe Armando et parle d'eux sans les citer. Le lecteur doit donc rechercher qui est le sujet de la diégèse. N1 recommence ensuite à tutoyer un de ses personnages (cette fois-ci Armando) mais sur tout un chapitre en alternance avec la troisième personne.

13 J'entends par « rassurant " le fait qu'il reprend une sorte de "norme" de narrateur établie depuis le roman réaliste du XIXème siècle.

14 «Tú, Andrés Choz, abuelo incestuoso, viejo verde lleno de lúbricos pensamientos, contemplas la forma de las peñas semejantes a las cercanas redondeces. " (p. 80) [Et Toi, Andrés Choz, grand-père incestueux, vieux beau plein de pensées lubriques, tu contemples la forme des rochers semblable aux rondeurs toutes proches]. (il s'agit de la deuxième rencontre avec Teresa et $\mathrm{Choz}$ et ils sont sur la plage. C'est moi qui souligne). 
Parallèlement à ces dédoublements de points de vues et de voix, Merino mêle à dessein le style direct, indirect et indirect libre. Comme pour l'utilisation des personnes grammaticales, la complexité va croissante au fur et à mesure du roman. $\mathrm{Si}$ au début, le style direct clairement marqué prédomine, il se transforme vite en style direct non marqué typographiquement :

"Andrés Choz se acerca a ellos: Viva la juventud exclama $»^{15}$, (p. 73)

puis en style indirect libre qui joue en même temps avec les personnes :

« No, no se mareaba mucho, repuso Andrés Choz, o qué sé yo, hace tanto tiempo que no subo a un barco que ni lo recuerdo, $i$ dónde está tu amigo ? Y la muchacha se le quedó mirando. »16 (p. 75)

Ces deux procédés atteignent leur paroxysme dans le dernier chapitre du roman. Il s'ouvre sur N2 qui est ensuite relayé par N1 qui tutoie Choz. L'alternance est marquée typographiquement jusqu'à la page 246 où un personnage du récit-cadre (Julia, la femme défunte de Choz) fait une incursion dans le récit inséré17. Le style indirect libre contribue à opérer la fusion entre $\mathrm{N} 1$ et $\mathrm{A} 1$ dans les dernières phrases du roman :

«Quedan cuatro meses hasta fin de año y luego quién sabe.

Estará ya en las pasiones de la especie humana, será uno de ellos.

15 [Andrés Choz s'approche d'eux : Vive la jeunesse s'exclame-til].

16 [Non, il n'avait pas trop le mal de mer, répondit Andrés Choz, ou je ne sais pas, il y a tellement longtemps que je suis monté sur un bateau, que je ne m'en rappelle même pas, où est ton ami ? Et la jeune fille le regarda fixement].

17 «Julia insinuaba que podría ser como un muchacho de diez o doce años, y justificar su falta de conocimiento y recuerdos con una amnesia, por ejemplo, .... » (p. 246) [Julia insinuait qu'il pourrait être comme un enfant de dix ou douze ans, et justifier son manque de connaissance et de souvenirs avec une amnésie, par exemple,...] 
Mañana llamo a Gordo y se lo digo.

Hay que dejar la novela mucho mejor. Por lo menos, poder decir ahí queda eso.» ${ }^{18}$ (p. 251)

Nous ne savons plus qui de $\mathrm{N} 1$ ou de Al prononce ces derniers mots.

Par conséquent la polyphonie narrative, doublée du style indirect libre, conduit à dupliquer le narrateur jusqu'à le confondre avec son personnage, ou à en faire son double, comme une image dans un miroir. Avant de voir quels en sont les enjeux, il nous faut montrer brièvement en quoi les personnages et le discours littéraire constituent des mises en abyme.

El Gordo, l'éditeur de Choz et destinataire des lettres de celui-ci, est le principal récepteur du roman de Ons. En ce sens, il est le miroir du lecteur du roman et ce que Choz lui dit le pousse à réfléchir (et oblige le lecteur à en faire autant) sur ce qui est déjà écrit et sur ce qui va s'écrire. Il est donc récepteur mais aussi critique du texte de $\mathrm{Choz}$ et conseiller de celui-ci ${ }^{19}$. Le personnage d'Armando, le jeune thésard en lettres constitue une autre mise en abyme du critique littéraire. Il n'a cependant qu'une place secondaire et n'est en réalité qu'un prétexte pour permettre à $\mathrm{Choz}$ d'exposer ses théories sur la littérature 20 .

C'est bien évidemment Andrés Choz, en tant qu'écrivain, qui est le moteur principal de la réflexivité dans le livre. Outre des commentaires stylistiques et des précisions sous forme de commentaire quant à ses intentions d'auteur, il montre surtout le travail de l'écrivain. L'exemple le plus

18 [Il reste quatre mois avant la fin de l'année et ensuite, qui sait ? Il sera déjà dans les passions de l'espèce humaine, il sera l'un d'entre eux. / Demain j'appelle Gordo et je le lui dis. Il faut beaucoup améliorer le roman. Du moins, pouvoir dire que tout s'arrête là].

19 Ces critiques sont connues du lecteur par la voix de Choz dans ces lettres. Par exemple : «En cuanto a tu carta, confieso que me ha dejado perplejo, sobre todo cuando dices que debo pormenorizar las circunstancias del accidente. " (p. 160) [Quant à ta lettre, j'avoue qu'elle m'a laissé perplexe, surtout quand tu dis que je dois détailler les circonstances de l'accident].

${ }^{20}$ On le voit notamment au chapitre «(Tres)» (pp. 69-80). 
probant est certainement celui du miroir qui reflète les premières pages du livre écrit par $\mathrm{Choz}$ :

«En el espejo del lavabo se refleja la superficie de la mesa camilla con la máquina de escribir y el Capítulo Primero. ${ }^{21}$ (p. 53)

Le lecteur a déjà pris connaissance de ce chapitre avant cette référence 22 . Cependant l'ordre initial des chapitres est remis en cause un peu plus loin dans une note marginale de $\mathrm{Choz}$ au début du « CAPÍTULO SEGUNDO »:

« (Podría ser Introducción o Capítulo Primero. Entonces, el actual Cap. $1^{\circ}$ vendría a continuación). $»^{23}$ (p. 61)

C'est donc le procédé d'écriture qui nous est montré ici et qui nous oblige à reconstruire le roman, comme si nous étions nous-mêmes son auteur et devenions ainsi une de ses voix narratives. La présence du miroir est loin d'être anodine puisqu'elle nous renvoie notre propre image en même temps que celle de $\mathrm{Choz}$ et fait ainsi de celui-ci le double de Merino lui-même.

José María Merino nous propose par conséquent un jeu savant dans lequel le statut du narrateur est remis en cause parce qu'il est dédoublé grâce notamment à la typographie et à l'utilisation du style indirect libre. Cette démultiplication des voix narratives met en place un jeu de miroirs qui brouille les rapports entre réalité et fiction. Qui de Choz ou de Ons a créé l'autre? A la fin du roman, le récit-cadre et le récit inséré sont fortement imbriqués à tel point qu'ils ne font plus qu'un et que Julia (personnage de la réalité de Choz) est citée dans le récit inséré. S'opère alors une inversion des rôles : le récit inséré serait en fait le récit-cadre et Ons le créateur de Choz et non sa créature. Merino s'interroge donc sur le rôle et la place du narrateur et sur l'existence des personnages littéraires. Il

21 [La surface de la table de chevet avec la machine à écrire et le Chapitre Un se reflète dans le miroir des toilettes]

22 Aux pages 39 à 49 du roman.

23 [(Pourrait être Introduction ou Chapitre Un. L'actuel Chap. 1 viendrait ensuite)] 
commence à poser à la fin de ce roman la question qui constituera le sujet central de La orilla oscura ${ }^{24}$ : est-ce la réalité qui influence la fiction ou l'inverse?

24 Il s'agit du troisième roman de Merino, publié en 1985. 


\section{BIBLIOGRAPHIE}

CANDAu (A.) : La obra narrativa de José María Merino, Diputación Provincial de León, 1992.

DÄLLENBACH (L.) : Le récit spéculaire. Essai sur la mise en abyme, Collection Poétique, Seuil, Paris, 1977.

Dotras (A.-M.) : La novela española de metaficción, Ensayos Júcar, Madrid, 1994.

Genette (G.) : Figures III, Collection Poétique, Seuil, Paris, 1972.

Genette (G.) : Palimpsestes. La littérature du second degré, Points Essais n 257 , Seuil, Paris, 1982.

MARTÍNEZ CACHERo (J.-M.) : La novela española entre 1936 y el fin del siglo. Historia de una aventura, Collection "Literatura y Sociedad", Editorial Castalia, Madrid, 1997. 